\title{
Effect of Patellar Thickness on Knee Flexion in Total Knee Arthroplasty: A Biomechanical and Experimental Study
}

\author{
Mansour Abolghasemian, MD ${ }^{a}$, Saeid Samiezadeh, PhD Candidate ${ }^{b}$, Amir Sternheim, MD ${ }^{a}$, \\ Habiba Bougherara, PhD, PEng ${ }^{\mathrm{b}}$, C. Lowry Barnes, MD $^{\mathrm{c}}$, David J. Backstein, MD, FRCSC ${ }^{\mathrm{d}}$ \\ a Clinical Fellow of Arthroplasty in the Division of Orthopaedic Surgery, Mount Sinai Hospital, University of Toronto, Toronto, ON, Canada \\ ${ }^{\mathrm{b}}$ Department of Mechanical and Industrial Engineering, Ryerson University, Toronto, ON, Canada \\ c University of Arkansas for Medical Sciences, Little Rock, AR, USA \\ d Head of the Division of Orthopedic Surgery, Mount Sinai Hospital, University of Toronto, Toronto, ON, Canada
}

\section{A R T I C L E I N F O}

\section{Article history:}

Received 6 February 2013

Accepted 15 April 2013

\section{Keywords:}

knee arthroplasty

knee flexion

patellar thickness

range of motion

over-stuffing

\begin{abstract}
A B S T R A C T
A biomechanical computer-based model was developed to simulate the influence of patellar thickness on passive knee flexion after arthroplasty. Using the computer model of a single-radius, PCL-sacrificing knee prosthesis, a range of patella-implant composite thicknesses was simulated. The biomechanical model was then replicated using two cadaveric knees. A patellar-thickness range of $15 \mathrm{~mm}$ was applied to each of the knees. Knee flexion was found to decrease exponentially with increased patellar thickness in both the biomechanical and experimental studies. Importantly, this flexion loss followed an exponential pattern with higher patellar thicknesses in both studies. In order to avoid adverse biomechanical and functional consequences, it is recommended to restore patellar thickness to that of the native knee during total knee arthroplasty.
\end{abstract}

(C) 2014 The Authors. Published by Elsevier Inc. Open access under CC BY-NC-SA license.
Total knee arthroplasty (TKA) is a common and generally successful operation. Numerous studies have analyzed surgical techniques and factors influencing its short-term and long-term success [1-4]. The functional outcome and survivorship after TKA are two primary factors often utilized to measure the success of the procedure. A comprehensive relationship between the biomechanical features of TKA (e.g. knee alignment, component position and size) and its function and longevity has been long recognized. Knee range of motion, as a major functional index, has been reported by several studies to influence many daily activities and patient satisfaction [5-8].

The function of the patello-femoral articulation is known to have a significant impact on the outcome of the TKA procedure [9]. Despite the controversy regarding the use of patellar resurfacing, this technique is still common in total knee arthroplasty [10]. Employing the proper technique when resurfacing the patella, is essential to avoid over-stuffing and mal-tracking which can result in anterior knee pain and sub-optimal range of motion. Yet, there is no consensus on

The Conflict of Interest statement associated with this article can be found at http:// dx.doi.org/10.1016/j.arth.2013.04.026.

Reprint requests: Saeid Samiezadeh, PhD Candidate, Department of Mechanical and Industrial Engineering, Ryerson University, Toronto, ON, Canada, M5B 2K3. the exact relationship between the patella-implant thickness and the biomechanical function (including range of motion) of the knee after TKA [11]. The purpose of the present biomechanical and experimental study is therefore, to determine and analyze the relationship between patellar thickness and range of motion after TKA, and to identify the factors influencing this relationship.

\section{Materials and Methods}

This study consisted of two parts: a computer-based biomechanical study and an experimental analysis using cadaveric knees in order to validate the biomechanical study. This study started after institutional review board approval.

\section{Biomechanical Study}

A biomechanical model of the human knee was made in SolidWorks software (Dassault Systèmes SolidWorks Corp, MA) based on data from previous studies [12,13]. The model consisted of the Quadriceps muscles, patella, patella ligament, femur and tibia. Virtual 3-dimensional (3D) total knee arthroplasty was performed using a computer model for the Evolution Knee Replacement System (Wright Medical Inc., Arlington, Tenn). A two dimensional (2D) model was then developed in the sagittal plane where the tracking of the patella was investigated. Since the passive knee flexion was 
investigated in this study, the muscles, ligaments, and the patella were replaced by lines with constant lengths. This knee system was selected because of its single radius of curvature which makes it suitable for computational studies. Fig. 1 shows the details of the biomechanical model.

The model was then used to obtain the maximum possible flexion as a function of patellar thickness, assuming that a constant flexion force is applied to the knee which is mainly due to its weight. This force was sufficient in all different conditions to stretch the quadriceps mechanism to a certain amount of excursion. A range of $15 \mathrm{~mm}$ to $39 \mathrm{~mm}$ in patellar thickness with $1 \mathrm{~mm}$ increments was assumed and the resulting passive knee flexions were obtained. This seemingly excessively wide range of patellar thicknesses was studied to magnify the behavior pattern of the knee in response to the patellar thickness changes.

The model took into account the effect of the joint geometry and the length of the muscles, tendons, and ligaments was considered constant due to the assumption of a constant excursion. The distance $l$ is introduced as $l=t+d$, in which $d$ is the distance between the anterior border of the patella and the poly and $t$ is the poly thickness which was changed in different stages. The angle $\theta$, representing the supplementary flexion angle, is defined as the angle between the Quadriceps muscles and the tibia as depicted in Fig. 1.

The effects of size and radius of curvature of the patellar polyethylene component were also investigated. For this purpose, three different combinations of polyethylene sizes and thicknesses were studied.

\section{Experimental Investigation}

The authors utilized two cadaveric knees of thin males with anatomically intact joints and full range of motion (ROM). Using a standard medial parapatellar approach, a cemented posterior stabilized (PS) TKA (Evolution Knee Replacement System, Wright Medical Inc., Arlington, Tenn, USA) was implanted on the right knee of each cadaver. A total of $10 \mathrm{~mm}$ of bone and articular cartilage was removed from each patella. Patellar prosthesis trials with variable thicknesses ranging from nine to $24 \mathrm{~mm}$ with $3 \mathrm{~mm}$ increments were implanted sequentially. This range of implant thicknesses led to an incremental increase of patellar bone-prosthesis composites from one millimeter less than the original patellar thickness to up to $14 \mathrm{~mm}$ thicker than the original thickness of the patella. This wide range of patellar thickness was applied to investigate the knee behavior accurately.

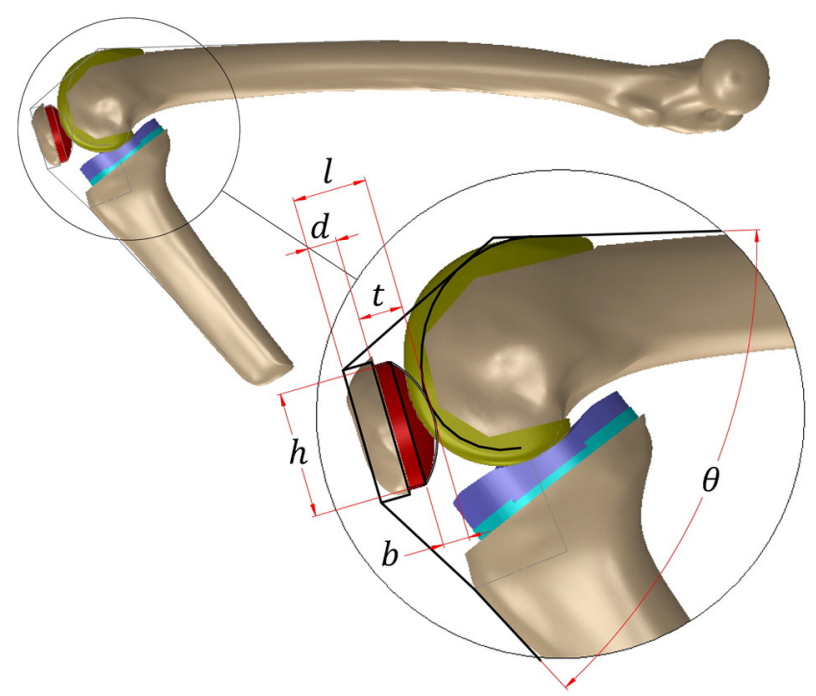

Fig. 1. The biomechanical knee model, $\theta$ represents the supplementary angle of knee flexion, $l$ represents the distance between patella groove and the anterior border of the patella, $t$ is the poly thickness, $h$ is the poly size, and $b$ is the height of poly cone.

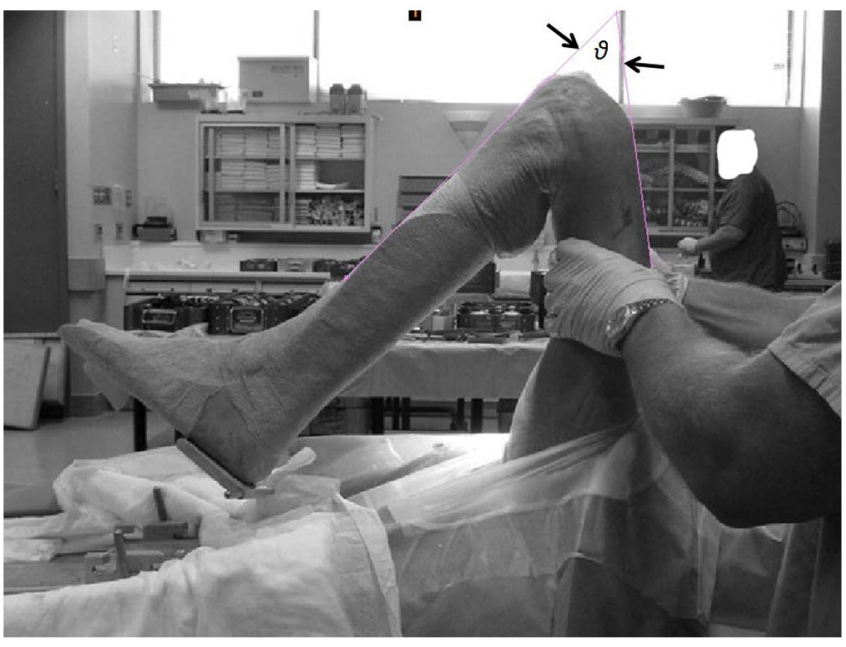

Fig. 2. Experimental setup: showing a typical flexion angle measurement for a specific patellar thickness. Note that $\theta$ was measured instead of $\alpha$ for convenience.

With each successive patellar implant, the hip was held in $90^{\circ}$ of flexion and neutral rotation. The knee was then allowed to bend passively by gravity alone without an additional force. This ensured that the flexion force applied to the knee remained constant in all trials. Using a fixed camera, photography of each trial was undertaken. Since the line of the quadriceps muscle, not the femur, was the reference in the biomechanical study, photography was assumed to be more accurate than radiography in identifying the direction of quadriceps. Subsequently, the angle of knee flexion was measured using Mimics software (Materialise, Leuven, Belgium) for each of the 12 pictures. Fig. 2 shows a typical measurement based on the photograph. The anterior border of the thigh was selected to represent the direction of quadriceps tendon and anterior border of the leg represented the direction of tibia. Angle $\theta$, as the supplementary flexion angle, was measured for convenience and the flexion angle $\alpha$ was obtained using the simple equation $\alpha=180-\theta$.

\section{Statistical Analysis}

The significance of the changes in knee flexion was analyzed using paired T test, with a $P$ value less than 0.05 deemed as significant.

\section{Results}

\section{Biomechanical Investigation}

For a patellar thickness spectrum ranging from $15 \mathrm{~mm}$ to $39 \mathrm{~mm}$, maximal flexion angle changed from $149.6^{\circ}$ to $95.9^{\circ}$ corresponding to an average flexion loss of $2.16^{\circ} / \mathrm{mm}$ of increased patellar thickness (Table 1). Interestingly, this change did not follow a linear pattern and in higher thicknesses of patellae, the flexion loss was increasingly

Table 1

Knee Flexion Angle and Flexion-Change Values in Biomechanical Study.

\begin{tabular}{lcc}
\hline Poly Thickness $(\text { Size } 26 \mathrm{~mm})^{\mathrm{a}}$ & Knee Flexion $(\alpha)$ & Flexion Loss/mm Thickness \\
\hline 3 & 149.65 & 1.32 \\
6 & 145.26 & 1.53 \\
9 & 140.19 & 1.78 \\
12 & 134.24 & 2.10 \\
15 & 127.12 & 2.52 \\
18 & 119.22 & 2.48 \\
21 & 111.26 & 2.69 \\
24 & 103.35 & 2.58 \\
27 & 95.90 & 2.41 \\
\hline
\end{tabular}

${ }^{\text {a }}$ For conciseness, patellar thicknesses have been presented in $3 \mathrm{~mm}$ increments. 


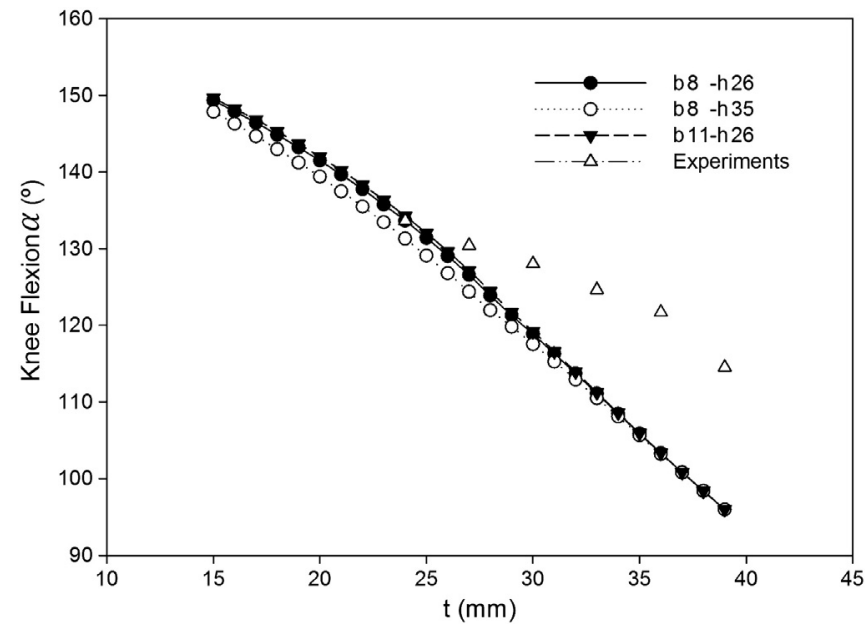

Graph 1. Biomechanical and experimental results of the degree of knee flexion as a function of patellar thickness. Three different polyethylene curvatures are included. For the experimental investigation, the knee flexion is the average of the two cadavers. $b$ and $h$ represent the height of poly cone and poly size, respectively.

larger than in lower thicknesses, as reflected in the flexion curve (Graph 1). For the first millimeter of thickness increase, from $15 \mathrm{~mm}$ to $16 \mathrm{~mm}$, the calculated flexion decreased by $1.32^{\circ}$. For the last incremental increase from $38 \mathrm{~mm}$ to $39 \mathrm{~mm}$, the change was $2.41^{\circ}$. Of note, the flexion-change curve had a notch in the higher range of thickness which was related to the polyethylene curvature. For more intense curvatures (smaller radii of curvature) the notch was sharper. Graph 2 illustrates the flexion-change curves when three different polyethylene sizes and curvatures were applied to the model.

\section{Cadaveric Investigation}

Similar to the biomechanical analysis, there was an obvious and steady decrease in knee flexion with the increase of patellar component thickness. The average flexion angle decreased from $133.66^{\circ}$ to $114.46^{\circ}\left(19.2^{\circ}\right)$ with a $15 \mathrm{~mm}$ increase in patellar prosthesis thickness. The mean amount of flexion loss for each millimeter of thicker patella was $1.28^{\circ}$. A non-linear pattern of flexion loss was observed again similar to the biomechanical study. When the patellar component thickness increased from 9 to $12 \mathrm{~mm}$ (which

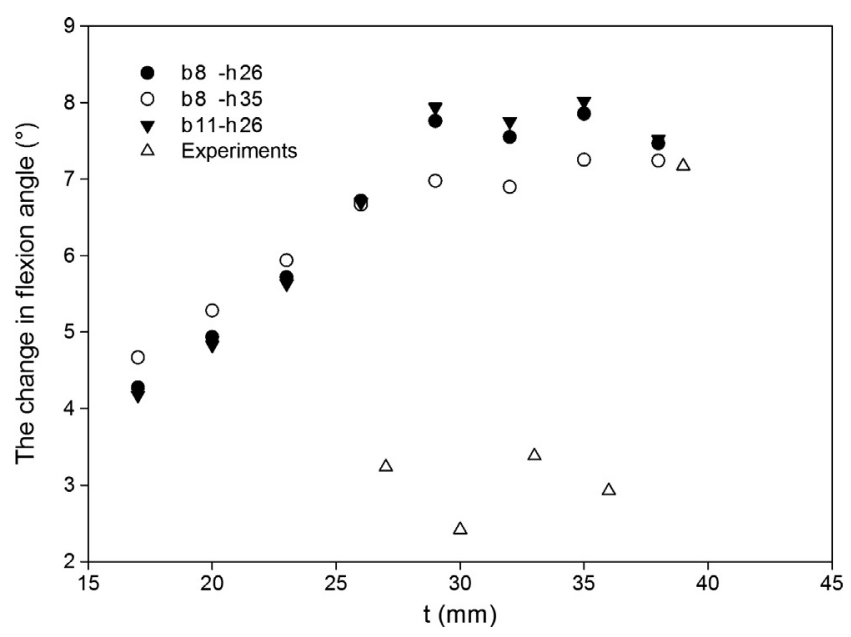

Graph 2. The change in flexion angle for different patellar thickness. Results of the biomechanical and experimental studies, in $3 \mathrm{~mm}$ increments of patellar thickness. Three different polyethylene curvatures are included. The largest patellar implant that has the least intense curvature created the shallowest notch. $b$ represents the height of poly cone and $h$ is the poly size. corresponded to a composite thickness of two millimeters more than the native patellar thickness) the average knee flexion angle decreased by $1.08^{\circ} / \mathrm{mm}$, but changing the thickness from 21 to $24 \mathrm{~mm}$ resulted in $2.53^{\circ} / \mathrm{mm}$ flexion loss. The different knee flexion angles obtained from the cadaveric investigation are tabulated in Table 2 .

Statistical analysis revealed that the change in flexion angles caused by increasing patellar thickness was statistically significant $(P$ value $=0.0022)$. The average amount of flexion loss for each $3 \mathrm{~mm}$ of increase in patellar thickness was 3.84 (95\% CI, 1.78 to 5.89 ).

\section{Discussion}

The results of this study suggest that increasing the thickness of patella causes an exponential loss of the knee flexion. Although factors influencing the knee range of motion following arthroplasty are numerous and some are not controllable by surgeons, the mechanical freedom of the knee in flexion is a basic requirement [14-17]. This is partially determined by the yield of knee extensor mechanism in which patellar thickness plays a role.

The impact of patellar thickness on the tibio-femoral kinematics has not been conclusively verified. Hsu et al in a study on 7 cadavers assessed the effect of three different patellar thicknesses, $2 \mathrm{~mm}$ thinner, the same thickness and $2 \mathrm{~mm}$ thicker than the native patella [18]. The authors did not appreciate any knee flexion loss with thicker patellae. They did not comment on whether they used posterior-stabilized or cruciate-retaining knee prosthesis type. Conversely, Bengs et al reported their results on the knee flexion of 31 cruciate retaining TKAs with four different patellar thicknesses, increased in two-millimeter increment [19]. They found a reverse correlation between knee flexion and the patellar thickness. The average amount of flexion loss with each one-millimeter increase in patellar thickness was found to be $1.5^{\circ}$. In contrast, several other clinical studies did not find any relationship between patellar thickness and knee flexion in follow-up measurements [20,21]. Some of these studies, however, did not consider the preoperative patellar thickness and therefore were not powered to comment on the effect of patellar thickness change on the knee flexion [20]. We feel that owing to the large variety of factors influencing knee range of motion following TKA, clinical studies might have been too confounded to be capable of showing the proper effect of patellar thickness on the range of motion. Assessing the physical constraint rendered to the knee with higher thicknesses of patella with usage of a computer model was deemed helpful in order to avoid confounders. Using a range of patellar thicknesses on a single knee during cadaveric studies also had the advantage of removing other factors affecting knee range of motion and showing the pure effect of patellar thickness.

The current study attempts to determine a comprehensive relationship between patellar thickness and knee range of flexion, based on the biomechanical limitations of the knee joint complex. The results were validated by similar findings in the cadaveric study. Both the biomechanical and the cadaveric studies demonstrated loss of flexion with increasing patellar thickness that was exponential with higher patellar thicknesses. The biomechanical study also showed that the curve of flexion-change per unit of increased patellar thickness had a notch. The point of maximal change in flexion before the notch was when the highest point of the patellar polyethylene came to contact with the femoral trochlea. After that, the flexion-change per unit of patellar thickness increase decreased by an amount that was related to the curvature of the patellar polyethylene. Smaller sizes of patella prosthesis have a smaller radius of curvature with the same thickness and lead to a sharper up-going flexion-change curve followed by a deeper notch. Conversely, bigger patellar prostheses followed a smoother pattern of flexion change. After the notch, the curve redirects upward, continuing to show exponential knee flexion loss. A similar notch was present in the curve of the cadaveric study. 
Table 2

Knee Flexion Angles in Cadaveric Study.

\begin{tabular}{|c|c|c|c|c|c|c|}
\hline & 9 mm Poly & 12 mm Poly & $15 \mathrm{~mm}$ Poly & $18 \mathrm{~mm}$ Poly & $21 \mathrm{~mm}$ Poly & $24 \mathrm{~mm}$ Poly \\
\hline Knee A Flexion & $133.67^{\circ}$ & $130.69^{\circ}$ & $127.71^{\circ}$ & $123.15^{\circ}$ & $117.77^{\circ}$ & $113.36^{\circ}$ \\
\hline Knee B Flexion & $133.64^{\circ}$ & $130.15^{\circ}$ & $128.36^{\circ}$ & $126.61^{\circ}$ & $126.34^{\circ}$ & $115.57^{\circ}$ \\
\hline Average & $133.66^{\circ}$ & $130.42^{\circ}$ & $128.04^{\circ}$ & $124.88^{\circ}$ & $122.05^{\circ}$ & $114.46^{\circ}$ \\
\hline
\end{tabular}

Whether this notch represented the same phenomenon was not confirmed due to an insufficient range of patellar thicknesses studied.

The adverse effect of decreased knee flexion cannot be overemphasized. This is of particular importance since there is adequate evidence in the literature indicating that higher range of flexion after knee arthroplasty is associated with greater satisfaction [22]. Moreover, increased patellar thickness may have other important clinical consequences as biomechanical studies have shown that increasing the thickness of patella-implant composite during a TKA increases the compression and shear forces on the patella-femoral joint $[18,23,24]$. Similarly, patellar tracking has been found to be related to patellar thickness [11,25]. Early loosening and shearing of the patellar component off the host patellar bone have been reported with thick patella-polyethylene composite [26]. On the other hand, an excessively thin patella may result in increased strain in the bone that makes it more susceptible to fracture and some authors have recommended against cutting the patella any thinner than 12 to $15 \mathrm{~mm}$ [23].

Based on the finding of this study a general recommendation can be made to cut the patella to a depth which restores the native patella's native thickness after resurfacing. This thickness is thought to provide the optimal kinetics for the patella, the implant and their interface $[16,21,22]$. To achieve this goal, one should take into consideration the thickness of lost cartilage, bone erosion and the thickness of the particular prosthetic implant being used. One limitation for such a practice is the original thickness of the native patella. Chmell et al have reported the average thickness of an arthritic patella to be $26.1 \mathrm{~mm}$ in male patients and $22.6 \mathrm{~mm}$ in female patients [27]. Jiang et al reported an average thickness of $21 \mathrm{~mm}$ in a population of Asian patients [28]. In patients with small bones, especially Asian females with thin native patella, preserving a traditional minimum thickness of $15 \mathrm{~mm}$ of patella bone for resurfacing will result in a composite that is thicker than the average original patella to some extent, which according to our results, may lead to adverse functional and biomechanical status of the knee. Very high patellar thicknesses after resurfacing, up to $41 \mathrm{~mm}$, have been reported due to inadequate technique $[26,28]$. Alternative approaches such as leaving the patella un-resurfaced, could be taken into consideration, if clinically appropriate.

\section{Clinical Implications}

One argument against the clinical application of the findings of this study is that in the cadaver, even with the thickest composite, the knee gained a flexion of $114^{\circ}$ which is not markedly less than the average amount of knee flexion after TKA in some reports [29]. This may indicate that patellar thickness does not play a role in the typical knee range of motion after TKA. This argument has two inherent problems. First, it ignores the impact of excessively thickened patellae in lowering the average range of motion of the knees in clinical studies. Second, in comparison to the average reported ROM, roughly half of TKAs gain higher flexion ranges which would not be possible with an over-thickened patella.

This study has some limitations including the small number of cadavers utilized for testing. Since the cadaveric part of the study was conducted for validation of the biomechanical analysis, two cadaveric knees were deemed sufficient by the authors. Second, both of the cadavers were thin males, leaving females or obese knees unexamined. Third, the accuracy of angle measurements would be improved if the limbs were rigidly fixed at $90^{\circ}$ of hip flexion. Analysis focused solely on the sagittal-plane movement of the patella that restricted our ability to predict the out-of-plane movements of the patella. Patellar movements in the coronal plane could accentuate the amount of flexion loss and alter the pattern of changes per unit of patellar thickness change. That may explain why the slope of the flexion change curve was less in the mathematical study compared to the experimental study. Furthermore, the effect of design, position and thickness of the prosthetic trochlea on overstuffing of the patellofemoral joint was not investigated in this study. The reciprocal influence of the two sides of the patellofemoral joint on its biomechanics could be the subject of a future study. Another shortcoming is that the results may differ in other knee arthroplasty systems, e.g. multiradius or cruciate retaining knee prostheses.

The study also has the following strengths. To our knowledge, this is the first combined biomechanical-experimental study on the relationship of patellar thickness and knee flexion pattern after TKA. A valid biomechanical model for knee kinematics was used and a wide range of patellar thicknesses was investigated. Validation of the results by the cadaveric investigation added to the reliability of the results as well as the clinical applicability.

In conclusion, this study shows that increased patellar thickness will lead to exponential flexion loss with its potential functional and biomechanical consequences. Caution is encouraged against adding to the original, native thickness of the patella when performing total knee arthroplasty and meticulous measurement of the cut is recommended to avoid this problem.

\section{Acknowledgments}

We thank Thomas Dorman and Wright Medical Technology Inc. for their unconditioned assistance in providing the requirements of this study.

\section{References}

1. Critchley RJ, Baker PN, Deehan DJ. Does surgical volume affect outcome after primary and revision knee arthroplasty? A systematic review of the literature. Knee 2012;19:513.

2. Kim YH, Kim JS, Choe JW, et al. Long-term comparison of fixed-bearing and mobilebearing total knee replacements in patients younger than fifty-one years of age with osteoarthritis. J Bone Joint Surg Am 2012;94:866

3. Ritter MA, Davis KE, Meding JB, et al. The effect of alignment and BMI on failure of total knee replacement. J Bone Joint Surg Am 2011;93:1588.

4. Shi MG, Lu HS, Guan ZP. Influence of preoperative range of motion on the early clinical outcome of total knee arthroplasty. Zhonghua Wai Ke Za Zhi 2006;44:1101.

5. Anouchi YS, Mcshane M, Kelly Jr F, et al. Range of motion in total knee replacement. Clin Orthop Relat Res 1996:87.

6. Dennis DA, Komistek RD, Stiehl JB, et al. Range of motion after total knee arthroplasty: the effect of implant design and weight-bearing conditions. J Arthroplasty 1998;13:748.

7. Maloney WJ, Schurman DJ. The effects of implant design on range of motion after total knee arthroplasty. Total condylar versus posterior stabilized total condylar designs. Clin Orthop Relat Res 1992:147.

8. Parsley BS, Engh GA, Dwyer KA. Preoperative flexion. Does it influence postoperative flexion after posterior-cruciate-retaining total knee arthroplasty? Clin Orthop Relat Res 1992:204.

9. Andriacchi TP, Stanwyck TS, Galante JO. Knee biomechanics and total knee replacement. J Arthroplasty 1986;1:211. 
10. Bargren JH, Blaha JD, Freeman MA. Alignment in total knee arthroplasty. Correlated biomechanical and clinical observations Clin Orthop Relat Res 1983:178.

11. Ghosh KM, Merican AM, Iranpour F, et al. The effect of overstuffing the patellofemoral joint on the extensor retinaculum of the knee. Knee Surg Sports Traumatol Arthrosc 2009;17:1211.

12. Wood DJ, Smith AJ, Collopy D, et al. Patellar resurfacing in total knee arthroplasty: a prospective, randomized trial. J Bone Joint Surg Am 2002;84-A:187.

13. Baldwin JL, House CK. Anatomic dimensions of the patella measured during total knee arthroplasty. J Arthroplasty 2005;20:250.

14. Steinbruck A, Milz S, Woiczinski M, et al. Anatomy and biomechanics of the patellofemoral joint: physiological conditions and changes after total knee arthroplasty. Orthopade 2011:40:848.

15. Harvey IA, Barry K, Kirby SP, et al. Factors affecting the range of movement of total knee arthroplasty. J Bone Joint Surg Br 1993;75:950

16. Kotani A, Yonekura A, Bourne RB. Factors influencing range of motion after contemporary total knee arthroplasty. J Arthroplasty 2005;20:850.

17. Ritter MA, Harty LD, Davis KE, et al. Predicting range of motion after total knee arthroplasty. Clustering, log-linear regression, and regression tree analysis. J Bone Joint Surg Am 2003;85-A:1278.

18. Hsu HC, Luo ZP, Rand JA, et al. Influence of patellar thickness on patellar tracking and patellofemoral contact characteristics after total knee arthroplasty. J Arthroplasty 1996;11:69.

19. Bengs BC, Scott RD. The effect of patellar thickness on intraoperative knee flexion and patellar tracking in total knee arthroplasty. J Arthroplasty 2006;21:650.
20. Gatha NM, Clarke HD, Fuchs R, et al. Factors affecting postoperative range of motion after total knee arthroplasty. J Knee Surg 2004;17:196.

21. Ritter MA, Pierce MJ, Zhou H, et al. Patellar complications (total knee arthroplasty). Effect of lateral release and thickness. Clin Orthop Relat Res 1999:149.

22. Devers BN, Conditt MA, Jamieson ML, et al. Does greater knee flexion increase patient function and satisfaction after total knee arthroplasty? J Arthroplasty 2011;26:178.

23. Reuben JD, Mcdonald CL, Woodard PL, et al. Effect of patella thickness on patella strain following total knee arthroplasty. J Arthroplasty 1991:6:251.

24. Oishi CS, Kaufman KR, Irby SE, et al. Effects of patellar thickness on compression and shear forces in total knee arthroplasty. Clin Orthop Relat Res 1996:283.

25. Youm YS, Cho WS, Woo JH, et al. The effect of patellar thickness changes on patellar tilt in total knee arthroplasty. Knee Surg Sports Traumatol Arthrosc 2010;18:923.

26. Singh VK, Singh PK, Singh Y, et al. Atraumatic patellar prosthesis dislocation with patellar tendon injury following a total knee arthroplasty: a case report. J Med Case Rep 2011;4:11.

27. Dinov ID, Rubin D, Lorensen W, et al. iTools: a framework for classification, categorization and integration of computational biology resources. PLoS One 2008;3:2265.

28. Jiang CC, Yip KM, Liu DH. Patellar thickness in total knee replacement. J Formos Med Assoc 1994;93:417.

29. Lizaur A, Marco L, Cebrian R. Preoperative factors influencing the range of movement after total knee arthroplasty for severe osteoarthritis. J Bone Joint Surg Br 1997;79:626. 